\title{
Review: selective serotonin reuptake inhibitors reduce symptoms in premenstrual syndrome
}

Dimmock PW, Wyatt KM, Jomes PW, et al. Efficacy of selective serotonin-reuptake inhibitors in premenstrual syndrome: a systematic review. Lancet 2000 Sep 30;356:1131-6.

\author{
QUESTION: In women with severe premenstrual syndrome (PMS), are selective \\ serotonin reuptake inhibitors (SSRIs) effective?
}

\section{Data sources}

Studies were identified by searching Medline (1966-99), EMBASE/Excerpta Medica (1988-98), PsycLIT (197497), CINAHL (1982-99), and the Cochrane Controlled Trials Register database; scanning the reference lists of identified articles; and contacting the manufacturers of SSRIs.

\section{Study selection}

Studies were selected if they were randomised, double blind, placebo controlled trials investigating SSRIs in the management of PMS.

\section{Data extraction}

Data were independently extracted in duplicate on study design, patient characteristics, drug type and dose, outcome measures (primary outcome measure was reduction in overall PMS symptoms), and side effects.

\section{Main results}

15 studies met the selection criteria and involved 904 women (570 allocated to active treatment and 435 allocated to placebo, including 101 in crossover trials). The 2 most studied SSRIs were fluoxetine (7 trials) and sertraline ( 5 trials). Most of the trials presented continuous data; thus, an overall standardised mean difference was calculated by using a random effects model. The overall standardised mean difference for reduction in PMS symptoms in favour of SSRIs was $-1.1(95 \%$ CI -1.4 to -0.8 ), which is equivalent to an odds ratio of 6.9 (CI 3.9 to 12.2). In 7 trials where data could be extracted for a comparison between physical and behavioural symptoms, SSRIs were found to be effective in treating both types with no statistically significant variance in the overall standardised mean differences. No difference in the effectiveness of SSRIs existed when comparing continuous and intermittent doses or between trials funded by pharmaceutical companies and those funded otherwise. Withdrawal from the studies because of side effects was 2.5 times greater (CI 1.6 to 3.7 ) in the SSRI group than in the placebo group.

\section{Conclusion}

In patients with severe premenstrual syndrome, selective serotonin reuptake inhibitors reduce symptoms.

\section{COMMENTARY}

The well done review by Dimmock $e t$ al shows that SSRIs improve symptoms in PMS. Diagnostic and Statistical Manual of Mental Disorders, Fourth Edition, criteria for the severe variant of PMS-premenstrual dysphoric disorder-are stringent and require prospective confirmation of the symptoms and their unique occurrence during the luteal phase and disappearance during the follicular phase for 2 consecutive cycles. Depression may occur concomitantly. No accepted pathophysiological abnormality exists, which means we have no diagnostic laboratory test for this difficult to define condition. It has, however, been shown that women with PMS exhibit an abnormal response to normal hormonal changes in contrast to those without PMS. ${ }^{1}$

As the authors have shown in a previous review, ${ }^{2}$ other pharmacological agents also work in PMS, which suggests that we try those with the lowest side effect profile after carefully identifying patients for treatment. But it would not be appropriate to limit treatment to medication with these often severely distressed patients. Easily deployed non-drug interventions also work well. Cognitive behavioural treatment is effective in patients with medically unexplained symptoms. ${ }^{3}$ Furthermore, careful attention to the providerpatient relationship in a patient-centred atmosphere has equally well documented benefits, as recent reviews note. ${ }^{4}$ Thus, one can best help symptomatic patients with a multidimensional intervention, one small part of which is an SSRI.

\section{Robert C Smith, MD Michigan State University East Lansing, Michigan, USA}

1 Schmidt PJ, Nieman LK, Danaceau MA, et al. Differential behavioral effects of gonadal steroids in women with and in those without premenstrual syndrome. $N$ Engl J Med 1998;338:209-16.

2 Wyatt KM, Dimmock PW, Jones PW, et al. Efficacy of vitamin B-6 in the treatment of premenstrual syndrome: systematic review. BMJ 1999;318:1375-81.

3 Sharpe M. Cognitive behavioural therapies in the treatment of functional somatic symptoms. In: Mayou R, Bass CM, Sharpe M, editors. Treatment of functional somatic symptoms. Oxford/New York: Oxford University Press, 1995:122-43.

4 Williams GC, Frankel RM, Campbell TL, et al. Research on relationship-centered care and healthcare outcomes from the Rochester biopsychosocial program: a selfdetermination theory integration. Families, Systems and Health 2000;18:79-90.

5 Stewart MA. Effective physician-patient communication and health outcomes: a review. CMAJ 1995;152:1423-33.
Source of funding: no external funding.

For correspondence: Dr P W Dimmock Academic Department of Obstetrics and Gynaecology, Keele University and North Staffordshire Hospital, Stoke on Trent ST4 6QG, UK. Fax +44(0)1782747319.

Abstract and commentary also appear in Evidence-Based Mental Health. 